\title{
Time evolution of open quantum many-body systems
}

\author{
Vincent R. Overbeck* and Hendrik Weimer \\ Institut für Theoretische Physik, Leibniz Universität Hannover, Appelstraße 2, 30167 Hannover, Germany
}

(Received 2 October 2015; published 11 January 2016)

\begin{abstract}
We establish a generic method to analyze the time evolution of open quantum many-body systems. Our approach is based on a variational integration of the quantum master equation describing the dynamics and naturally connects to a variational principle for its nonequilibrium steady state. We successfully apply our variational method to study dissipative Rydberg gases, finding very good quantitative agreement with small-scale simulations of the full quantum master equation. We observe that correlations related to non-Markovian behavior play a significant role during the relaxation dynamics towards the steady state. We further quantify this non-Markovianity and find it to be closely connected to an information-theoretical measure of quantum and classical correlations.
\end{abstract}

DOI: 10.1103/PhysRevA.93.012106

\section{INTRODUCTION}

Understanding the time evolution of quantum many-body systems is currently one of the most challenging tasks in both atomic and condensed matter physics, as concepts and tools developed for equilibrium systems are largely inapplicable. Open quantum systems exhibit even greater inherent complexity owing to the necessity to describe these systems in terms of statistical ensembles [1]. Here, we present a completely generic approach to analyze the time evolution of open quantum many-body systems, based on a variational principle.

The tremendous experimental progress in the manipulation of atomic quantum gases has allowed physicists to reach a regime where strong interactions can be combined with controlled dissipative processes [2-6], offering the potential to dissipatively prepare novel classes of quantum many-body states [7-9]. Recently, this experimental progress has been especially pronounced in the context of strongly interacting Rydberg gases [10-15], as the interaction and dissipation properties of Rydberg atoms can be widely tuned [16]. Consequently, dissipative Rydberg gases have emerged as an ideal environment to study strong interactions in an open quantum many-body system [17-27].

In this paper, we present a generic framework to investigate the time evolution of open quantum many-body systems. Our treatment naturally connects to a variational principle for the nonequilibrium steady state of the dynamics [28], and allows us to reduce the exponentially diverging number of degrees of freedom of the full time evolution to a small number of relevant parameters. We apply our method to the both experimentally and theoretically important problem of dissipative Rydberg gases and compare our results to small scale numerical simulations of the full quantum many-body problem. In our analysis, we find that the dynamics is inherently linked to the appearance of non-Markovian behavior within these systems, which persists even in the nonequilibrium steady state of the evolution. Remarkably, we observe that this non-Markovianity is closely related to an information-theoretical measure of the quantum and classical correlations present in the system.

\footnotetext{
*vincent.overbeck@itp.uni-hannover.de
}

\section{VARIATIONAL TREATMENT OF A RYDBERG GAS}

We consider the dynamics of open quantum systems, which is described in terms of a quantum master equation for the density operator $\rho$, according to a first order differential equation $d \rho / d t=\mathcal{L} \rho$, with the Liouvillian $\mathcal{L}$ being the generator of the dynamics. To be explicit, we focus on the case where the Liouvillian is given in Lindblad form, i.e.,

$$
\mathcal{L} \rho=-i[H, \rho]+\sum_{i}\left(c_{i} \rho c_{i}^{\dagger}-\frac{1}{2}\left\{c_{i}^{\dagger} c_{i}, \rho\right\}\right),
$$

where $H$ is the Hamilton-operator, the $c_{i}$ are the jump operators, describing the spontaneous decay of the Rydberg state. In the following, we will be interested in a numerical integration of the quantum master equation. Here, we will employ the implicit midpoint method [29]

$$
\rho(t+\tau)=\rho(t)+\frac{\tau}{2} \mathcal{L}[\rho(t)+\rho(t+\tau)]+O\left(\tau^{3}\right),
$$

where $t$ is the time and $\tau$ the integration step size, which we find to give the best tradeoff between accuracy of the integration and computational cost. Within our variational approach, we parametrize the density operator $\rho(t+\tau)$ by a set of variational parameters $\left\{\alpha_{i}\right\}$. Then, we find the variational solution by minimizing the variational norm $D$ given by

$$
D \equiv\left\|\rho(t+\tau)-\rho(t)-\frac{\tau}{2} \mathcal{L}[\rho(t)+\rho(t+\tau)]\right\|_{1} \rightarrow \min ,
$$

where $\|\cdot\|_{1}$ denotes the trace norm given by $\operatorname{Tr}\{|\cdot|\}$. Crucially, the state reached in the long time limit satisfying $\rho(t+\tau) \rightarrow \rho(t)$ corresponds to a direct variational principle for the nonequilibrium steady state [28]. We also note that our variational treatment is similar to recent approaches to treat the time evolution of one-dimensional systems based on matrix product states [30-32]. In higher dimensions, previous approaches to analyze the time evolution of open quantum many-body systems have largely been restricted to a mean-field decoupling [33-35]; however such a mean-field treatment is problematic for open systems [26,28,36-38].

In general, the computation of the variational solution is still an exponentially complex task. However, it is possible to obtain upper bounds to the variational solution, which can be calculated efficiently and are known to produce reliable results for the steady state $[28,36]$. As a basic example, let us 
consider a system consisting of $N$ identical two-level systems, interacting by a nearest-neighbor interaction. If we restrict our variational manifold to the set of product states, we may write the density operator as

$$
\rho^{p}=\prod_{i=1}^{N}\left(\frac{1}{2}+\sum_{\mu \in\{x, y, z\}} \alpha_{\mu} \sigma_{\mu}^{(i)}\right),
$$

where the $\sigma_{\mu}^{(i)}$ are the Pauli matrices. Then, we find an upper bound to the variational norm to be $[28,36]$

$$
D \leqslant \sum_{\langle i j\rangle}\left\|\rho_{i j}(t+\tau)-\rho_{i j}(t)-\tau \mathcal{L}\left[\rho_{i j}(t)+\rho_{i j}(t+\tau)\right]\right\|_{1},
$$

where $\rho_{i j}=\operatorname{Tr}_{i j j}\{\rho\}$ is the reduced two-site density operator of the particles $i$ and $j$. Variational states including nearestneighbor correlations can be expressed as

$$
\rho^{c}=\prod_{i=1}^{N} \rho_{i}+\sum_{\langle i j\rangle} \mathcal{R} C_{i j}+\sum_{\langle i j\rangle \neq\langle k l\rangle} \mathcal{R} C_{i j} C_{k l}+\cdots
$$

with the superoperator $\mathcal{R}$ transforming $1_{i}$ in $\rho_{i}$ and nearestneighbor correlations $C_{i j}$ defined as $\rho_{i j}=\rho_{i} \stackrel{\leftrightarrow}{\rho}_{j}+C_{i j}$. We can express the reduced density operator $\rho_{i j}$ in terms of the variational parameters as

$$
\rho_{i j}^{c}=\frac{1}{4}+\sum_{\mu, \nu} \alpha_{\mu \nu} \sigma_{\mu}^{(i)} \otimes \sigma_{\nu}^{(j)}
$$

In the case of such correlated variational states, the corresponding upper bound to the variational norm is found to be

$$
\begin{aligned}
D \leqslant & \sum_{\langle i j k\rangle} \| \rho_{i j k}(t+\tau)-\rho_{i j k}(t) \\
& -\tau \mathcal{L}\left[\rho_{i j k}(t)+\rho_{i j k}(t+\tau)\right] \|_{1},
\end{aligned}
$$

where $\rho_{i j k}=\operatorname{Tr}_{i / j k k}\{\rho\}$ is the reduced three-site density operator. In a translationally invariant system, the minimization reduces to a single three-site problem and can be solved with only a small number of variational parameters, even for infinitely large systems. The right hand side of Eq. (8) is a measure of the error made during the integration arising from the restriction of the dynamics to the variational manifold. In our analysis, this error is always the most important one, e.g., errors from the finite step size $\tau$ are negligible in comparison.

In the following, we will exemplify the application of this variational principle for the time evolution for open quantum systems by investigating dissipative Rydberg gases. To be specific, we consider a two-dimensional spin $1 / 2$ model, with the electronic ground state and a single Rydberg state corresponding to the two spin states. We neglect van der Waals interactions between the Rydberg states beyond the nearestneighbor distance. Then, we can express the Hamiltonian of the system as

$$
H=\frac{\Omega}{2} \sum_{i} \sigma_{x}^{(i)}+\frac{\Delta}{2} \sum_{i} \sigma_{z}^{(i)}+\frac{V}{4} \sum_{i j} \sigma_{z}^{(i)} \sigma_{z}^{(j)}
$$

where $\Omega$ and $\Delta$ follow from the laser parameters and $V$ is the interaction strength between neighboring sites. The dissipative terms in the Liouvillian are given in terms of jump operators of the form $c_{i}=\sqrt{\gamma} \sigma_{-}^{(i)}$, describing the spontaneous decay of the Rydberg state with a decay rate $\gamma$. In the following, we will

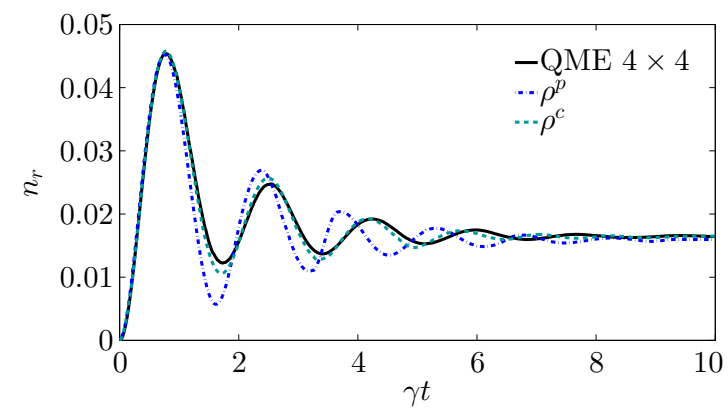

FIG. 1. Time evolution of the Rydberg density $n_{r}$ calculated via the quantum trajectory method in a $4 \times 4$ lattice (solid) and the variational methods including correlations $\rho^{c}$ (dashed) and product states $\rho^{p}$ (dotted) $(\Omega=\gamma, V=2 \gamma)$.

focus on the situation where the spin Hamiltonian describes an Ising model in a purely transverse field, i.e., $\Delta=0$.

The initial state is fully polarized into the electronic ground state, corresponding to the typical experimental situation. In the following, we compare the variational solutions based on product states and on states including nearest-neighbor correlations, to a quantum trajectory solution of the quantum master equation for a $4 \times 4$ lattice [39]. The resulting time evolution of the average Rydberg density $n_{r}$ is shown in Fig. 1. While the steady state value is consistent in all three approaches, the variational product state solution shows significant deviations during the relaxation dynamics. Remarkably, the variational approach based on correlated states shows much better quantitative agreement with the full numerical simulation for all times. This agreement is also found over a large region of both $\Omega$ and $V$; see the Appendix. Only in the regime with $\Omega \gg \gamma$ and $V \gg \gamma$ are there significant differences as the system undergoes a liquid-gas phase transition of the steady state [28]. However, in this regime, the quantum trajectories solution cannot be expected to provide an accurate description of the system in the thermodynamic limit, as strong finite size effects are present in a $4 \times 4$ system [36]. These results demonstrate that our variational approach can be used to accurately approximate the time evolution of open quantum many-body systems.

\section{NON-MARKOVIANITY AND QUANTUM LINEAR MUTUAL INFORMATION}

Using our variational method, we can now investigate the dynamics of the system in more detail. As the variational state involving two-site correlations provides an accurate description, we focus on the dynamics of the reduced twosite density operator $\rho_{i j}$ as our subsystem of interest. The interaction with the environment formed by the other sites results in additional coherent and dissipative terms. In the most general case, the equation of motion for the reduced density operator can be written in the form [40]

$$
\begin{aligned}
\frac{d}{d t} \rho_{i j}= & -i\left[H(t), \rho_{i j}\right] \\
& +\sum_{k=1}^{d^{2}-1} \gamma_{k}(t)\left(L_{k}(t) \rho_{i j} L_{k}^{\dagger}(t)-\frac{1}{2}\left\{L_{k}(t) L_{k}^{\dagger}(t), \rho_{i j}\right\}\right)
\end{aligned}
$$




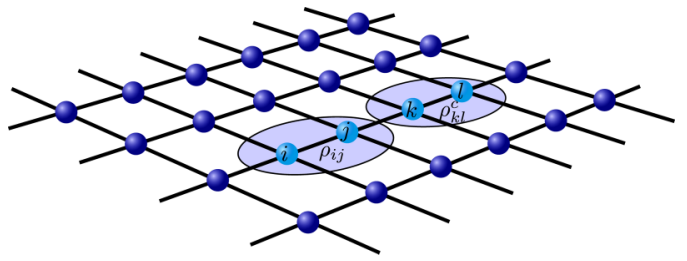

FIG. 2. Lattice structure for the calculation of the nonMarkovianity $f(t)$. The variational state $\rho_{i j}$ is being iterated over a complete set of initial states. The environment interacting with the sites $i$ and $j$ is given by the solution to the time evolution of the system for correlated states $\rho_{k l}^{c}$; see Fig. 1 .

where $d$ is the dimension of the state space, which in this case realizes to $d=4$, with the $L_{k}(t)$ forming an orthonormal basis according to

$$
\operatorname{Tr}\left[L_{k}(t)\right]=0, \quad \operatorname{Tr}\left[L_{j}^{\dagger}(t) L_{k}(t)\right]=\delta_{j k},
$$

and with the Hermitian operator $H(t)$ acting as an effective Hamiltonian. In contrast to the Liouvillian in the Lindblad form of Eq. (1), the generalized jump operators $L_{k}(t)$ can now become time dependent, and the generalized decay rates $\gamma_{k}(t)$ may become negative. The appearance of a negative decay rate corresponds to the dynamics being non-Markovian; conversely, if all decoherence rates are positive, the dynamics is Markovian. This relationship provides a natural measure for the degree of non-Markovianity of the dynamics,

$$
f(t)=\frac{1}{2} \sum_{k=1}^{d^{2}-1}\left[\left|\gamma_{k}(t)\right|-\gamma_{k}(t)\right],
$$

which is the sum of all negative decay rates [40]. This quantity is also closely related to other measures of non-Markovianity [41].

For the calculation of the generalized decay rates $\gamma_{k}(t)$, we consider the dynamics of a complete set of linearly independent states,

$$
\begin{aligned}
\rho^{00} & =\frac{\sigma_{0} \otimes \sigma_{0}}{4} ; \quad \rho^{m n}=\frac{1+\sigma_{m} \otimes \sigma_{n}}{4} \\
m, n & \in\{0, x, y, z\} ; m+n \neq 0,
\end{aligned}
$$

with $\sigma_{0}$ being the identity. While the reduced density operator $\rho_{i j}$ is iterated over a complete set, the state of the environment is fixed to the variational solution $\rho^{c}(t)$; see Fig. 2. This results in a consistent treatment of the non-Markovianity of the dynamics, which is governed by the correlations between the two-site system of interest and the environment formed by the remaining sites.

For the calculation of the non-Markovianity $f(t)$, we need to obtain both $\rho_{i j}(t)$ and $\dot{\rho}_{i j}(t)$. Here, we can readily compute $\rho_{i j}(t)$ using the variational method, from which we can determine its derivative according to

$$
\dot{\rho}_{i j}(t+\tau)=\frac{\rho_{i j}(t+2 \tau)-\rho_{i j}(t)}{2 \tau}+O\left(\tau^{3}\right) .
$$

The dynamics can be rewritten in the form

$$
\dot{\rho}_{i j}(t+\tau)=\sum_{\alpha \beta} c_{\alpha \beta}(t+\tau) G_{\alpha} \rho_{i j}(t+\tau) G_{\beta},
$$

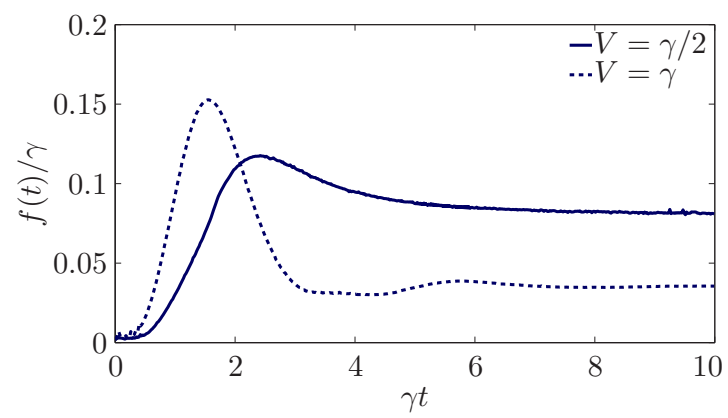

FIG. 3. Time evolution of the non-Markovianity $f(t)$ for an interaction strength of $V=\gamma / 2$ (solid) and $V=\gamma$ (dashed) $(\Omega=\gamma)$.

which is also valid in the case of non-Markovian dynamics [42]. Here, the $G_{\alpha}$ form a complete set of Hermitian operators, i.e., all possible combinations of the tensor product of two Pauli matrices. By iterating over all possible initial states for $\rho_{i j}$, we can uniquely determine the matrix elements $c_{\alpha \beta}(t)$, from which we can finally calculate the generalized decay rates $\gamma_{k}(t)$ [40]. Hence, the generalized decay rates and the effective jump operators $L_{k}(t)$ are calculated via the variational solution of the full quantum many-body model and differ from the purely Markovian jump operators in Eq. (1). Then, according to Eq. (12), we find that the non-Markovianity $f(t)$ is nonzero during the entire evolution; see Fig. 3. We also see that the degree of non-Markovianity is the largest for intermediate times, which explains why product states - which cannot generate non-Markovian dynamics-do not give an accurate description of the relaxation dynamics. Remarkably, the non-Markovianity even persists in the steady state.

Experimentally measuring the quantity $f(t)$ is a challenging task, requiring us to separate the dynamics of the two sites of interest from their environment. Therefore, we aim to construct a much more accessible quantity, which can serve as a witness for the non-Markovianity of the nonequilibrium steady state of the system. Here, we find that the quantum linear mutual information (QLMI), which depends only on the two-site density operator $\rho_{i j}$ fulfills this property. The QLMI is a measure for the quantum and classical correlations in the system and is defined as [43]

$$
I=S_{l}\left(\rho_{i} \otimes \rho_{j}\right)-S_{l}\left(\rho_{i j}\right),
$$

according to the linear entropy

$$
S_{l}\left(\rho_{i j}\right)=1-\operatorname{Tr}\left\{\rho_{i j}^{2}\right\} .
$$

Consequently, the QLMI is a natural extension of the (linear) entanglement entropy for mixed quantum states. As the QLMI is a functional of the reduced density operator $\rho_{i j}$, it can be experimentally determined using standard quantum state tomography. In our case, the relationship between the QLMI and non-Markovianity can be understood as both being intrinsically related to the correlations present in the system. Indeed, we observe a good quantitative agreement, up to a trivial constant factor, between the two measures; see Fig. 4.

Remarkably, this quantitative agreement is only found for the QLMI; it is absent for other measures such as the von Neumann mutual information $I_{\mathrm{VN}}$, where the linear entropy $S_{l}$ is replaced by the von Neumann entropy $S(\rho)=-\operatorname{Tr}\{\rho \ln \rho\}$. We can understand this property by considering an expansion 


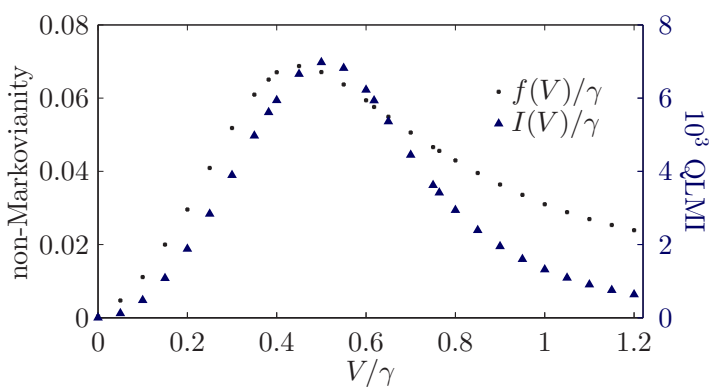

FIG. 4. Non-Markovianity $f(V)$ and the QLMI $I(V)$ of the steady state for different values of the interaction strength $V(\Omega=\gamma)$.

of $\rho_{i j}$ around product states,

$$
\rho_{i j}=\rho_{i} \otimes \rho_{j}+\varepsilon A,
$$

where $\varepsilon$ is the expansion parameter and $A=\sigma_{\kappa}^{(i)} \sigma_{\lambda}^{(j)}$ is a tensor product of two Pauli matrices. We now expand $\rho_{i}$ and $\rho_{j}$ in terms of the variational parameters $\alpha_{\mu}$, according to Eq. (4). Then, we find for the von Neumann mutual information

$$
I_{\mathrm{VN}}=\varepsilon \operatorname{Tr}\left\{A \ln \left(\rho_{i} \otimes \rho_{j}\right)\right\}+O\left(\varepsilon^{2}\right),
$$

which leads to a logarithmic dependence on the variational parameters $\alpha_{\mu}$. On the other hand, the non-Markovianity follows from minimizing the variational norm $D$. Here, we find that both $D$ and the variational solution for $\rho_{i j}(t+\tau)$ are a bilinear function in terms of the parameters $\alpha_{\mu}$, which is incompatible with the logarithmic dependence predicted by the von Neumann mutual information. In contrast, the leading term of the QLMI remarkably reproduces this bilinear form, i.e.,

$$
I=\varepsilon \alpha_{\kappa} \alpha_{\lambda}+O\left(\varepsilon^{2}\right) .
$$

This singles out the QLMI as the correct informationtheoretical measure to serve as a witness for non-Markovianity in our analysis. We also note that our focus on the linear contribution in $\varepsilon$ becomes less accurate for larger values of $V$ : In the case of sufficiently strong interactions, the coherent part of the dynamics essentially becomes frozen and the stationary state lies close to the pure state with all atoms being polarized into their electronic ground state. This is accompanied by a vanishing of the first order contribution to the QLMI, as all coefficients except for $\alpha_{z}$ approach zero. In this regime, the QLMI and the non-Markovianity indeed begin to deviate from each other; see Fig. 4, with the QLMI decaying faster than the non-Markovianity.

\section{SUMMARY}

In summary, we have successfully demonstrated a variational method for calculating the time evolution of open quantum many-body systems. Our method allows for a systematic treatment of correlations in the system, and thus gives access to evaluate quantities associated with non-Markovian dynamics. Finally, we wish to point out that our variational method is also applicable to the time evolution of closed quantum many-body systems in the absence of dissipation. Crucially, the description in terms of correlated variational states generically leads to an effective equation of motion that contains dissipative terms that are formed by tracing out the environment formed by the other sites of the system. We thus expect our method to be highly relevant for the investigation of quench dynamics [44-46], thermalization in closed quantum systems [47,48], or quantum control of many-body systems [49].

\section{ACKNOWLEDGMENTS}

We acknowledge fruitful discussions with O. Morsch and T. Osborne. This work was funded by the Volkswagen Foundation and the DFG Research Training Group 1729.
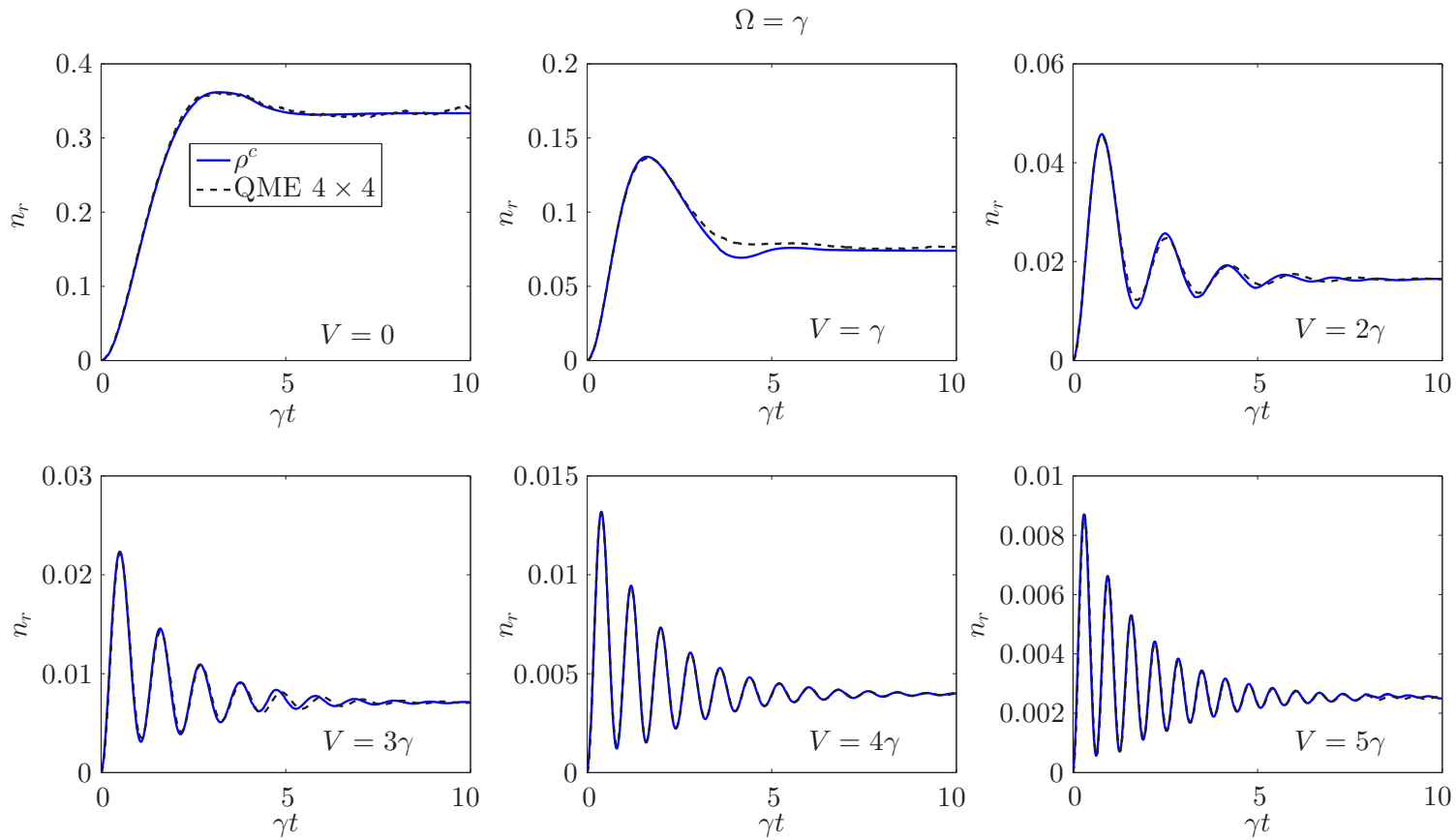

FIG. 5. Dynamics of the Rydberg density $n_{r}$ for $\Omega=\gamma$, calculated by the variational approach (solid) and the quantum trajectory method (dashed). 

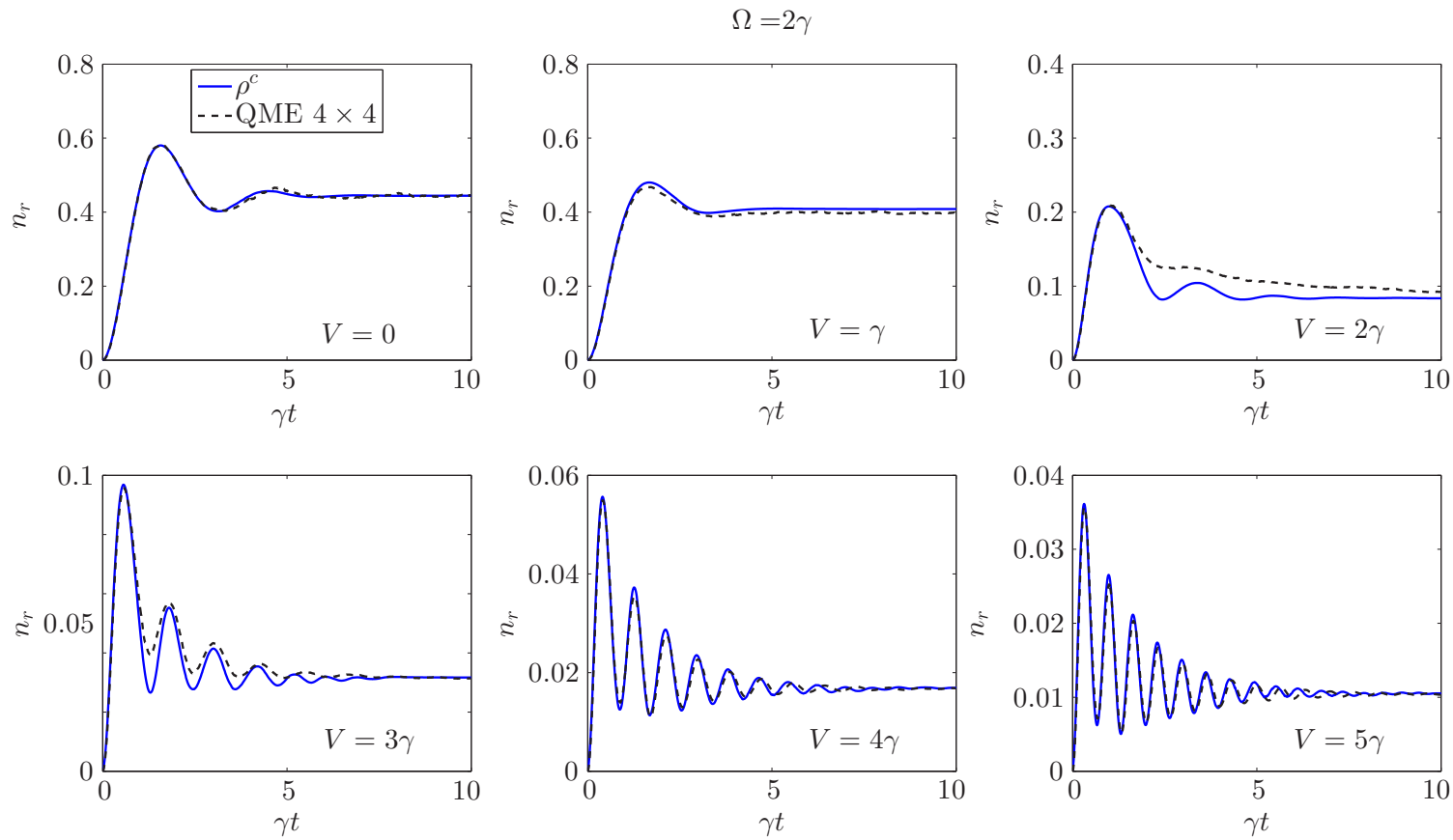

FIG. 6. Rydberg density $n_{r}$ for $\Omega=2 \gamma$ and several values of the interaction strength $V$.

APPENDIX: COMPARISON OF THE VARIATIONAL METHOD AND THE QUANTUM TRAJECTORY METHOD

In the following, we compare the dynamics gained via the variational principle with the dynamics of the full quantum master equation. The latter is calculated by the quantum trajectory method on a two-dimensional $4 \times 4$ lattice with periodic boundary conditions.
Figs. 5-8 show the dynamics of the Rydberg density for $\Omega=\gamma$ to $\Omega=4 \gamma$, respectively, for different interaction strengths $V$. For larger values of $\Omega$ and $V$, the system undergoes a phase transition of the nonequilibrium steady state [28]. In that region, the variational solution does not match very well with the solution of the full quantum master equation anymore, as can be seen for the case of $\Omega=3 \gamma$ and $\Omega=4 \gamma$. This can be traced back to two reasons:
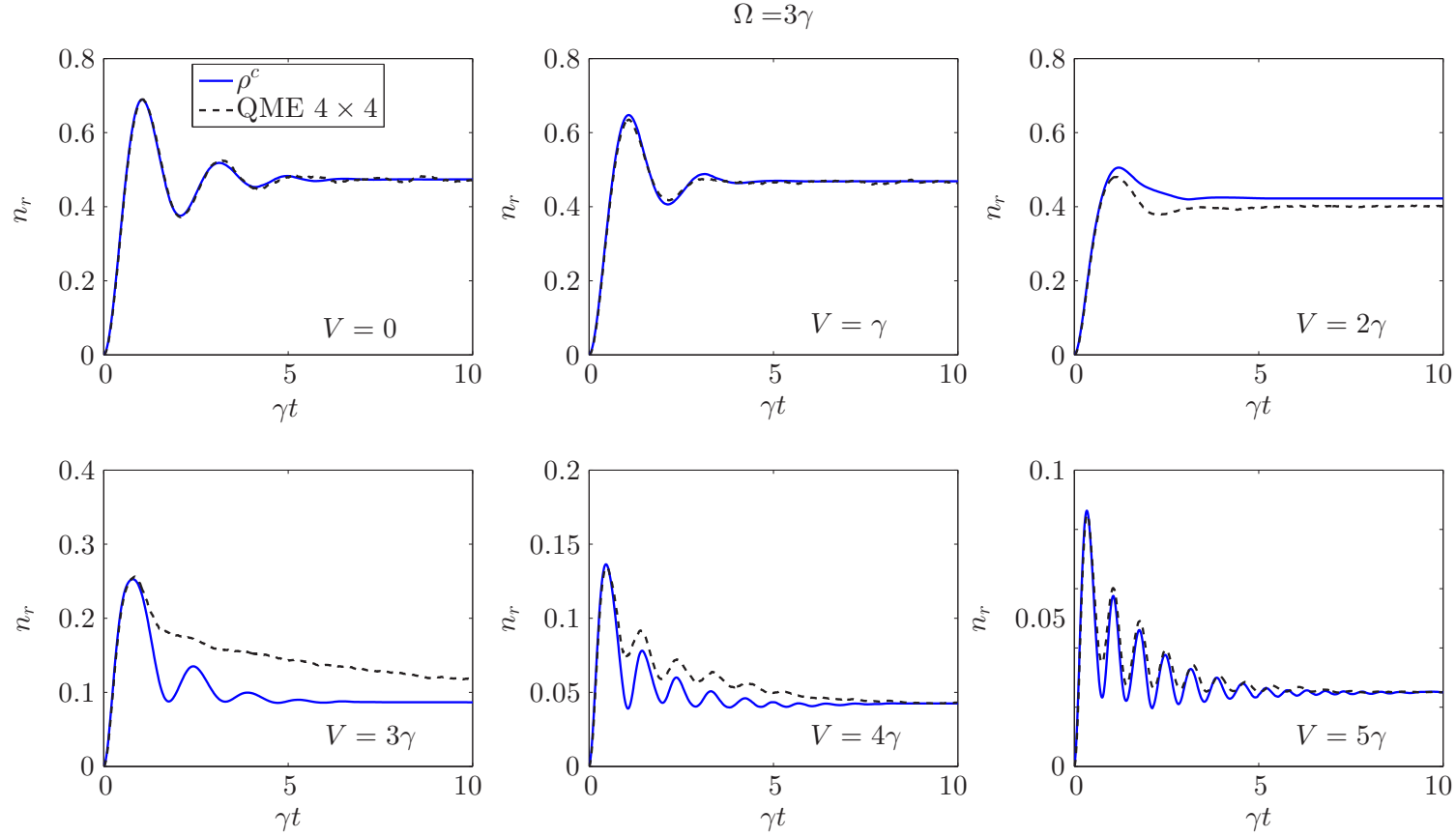

FIG. 7. Rydberg density for $\Omega=3 \gamma$ and several values of $V$. 

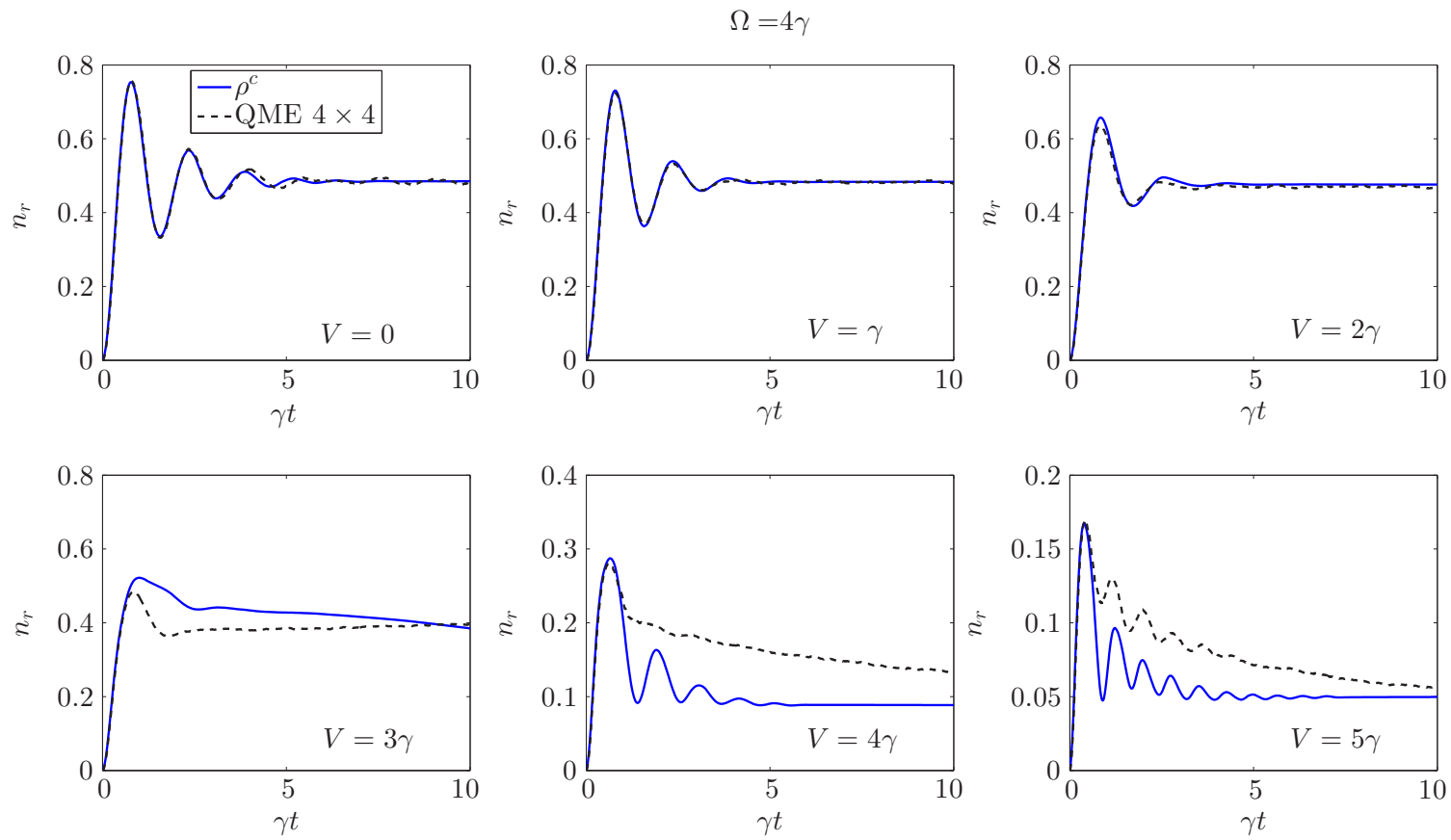

FIG. 8. Rydberg density for $\Omega=4 \gamma$ and several values of $V$.

(i) Close to the phase transitions, long-range correlations become relevant, whereas our variational approach only takes nearest-neighbor correlations into account. (ii) At the same time, the quantum trajectories solution is subject to finite size effects, which also become important close to the transition.
[1] H.-P. Breuer and F. Petruccione, The Theory of Open Quantum Systems (Oxford University Press, Oxford, 2002).

[2] N. Syassen, D. M. Bauer, M. Lettner, T. Volz, D. Dietze, J. J. García-Ripoll, J. I. Cirac, G. Rempe, and S. Dürr, Strong dissipation inhibits losses and induces correlations in cold molecular gases, Science 320, 1329 (2008).

[3] K. Baumann, C. Guerlin, F. Brennecke, and T. Esslinger, Dicke quantum phase transition with a superfluid gas in an optical cavity, Nature (London) 464, 1301 (2010).

[4] J. T. Barreiro, M. Müller, P. Schindler, D. Nigg, T. Monz, M. Chwalla, M. Hennrich, C. F. Roos, P. Zoller, and R. Blatt, An open-system quantum simulator with trapped ions, Nature (London) 470, 486 (2011).

[5] H. Krauter, C. A. Muschik, K. Jensen, W. Wasilewski, J. M. Petersen, J. I. Cirac, and E. S. Polzik, Entanglement Generated by Dissipation and Steady State Entanglement of Two Macroscopic Objects, Phys. Rev. Lett. 107, 080503 (2011).

[6] G. Barontini, R. Labouvie, F. Stubenrauch, A. Vogler, V. Guarrera, and H. Ott, Controlling the Dynamics of an Open Many-Body Quantum System with Localized Dissipation, Phys. Rev. Lett. 110, 035302 (2013).

[7] S. Diehl, A. Micheli, A. Kantian, B. Kraus, H. P. Büchler, and P. Zoller, Quantum states and phases in driven open quantum systems with cold atoms, Nat. Phys. 4, 878 (2008).

[8] F. Verstraete, M. M. Wolf, and J. Ignacio Cirac, Quantum computation and quantum-state engineering driven by dissipation, Nat. Phys. 5, 633 (2009).

[9] H. Weimer, M. Müller, I. Lesanovsky, P. Zoller, and H. P. Büchler, A Rydberg quantum simulator, Nat. Phys. 6, 382 (2010).
[10] U. Raitzsch, R. Heidemann, H. Weimer, B. Butscher, P. Kollmann, R. Löw, H. P. Büchler, and T. Pfau, Investigation of dephasing rates in an interacting Rydberg gas, New J. Phys. 11, 055014 (2009).

[11] C. Carr, R. Ritter, C. G. Wade, C. S. Adams, and K. J. Weatherill, Nonequilibrium Phase Transition in a Dilute Rydberg Ensemble, Phys. Rev. Lett. 111, 113901 (2013).

[12] N. Malossi, M. M. Valado, S. Scotto, P. Huillery, P. Pillet, D. Ciampini, E. Arimondo, and O. Morsch, Full Counting Statistics and Phase Diagram of a Dissipative Rydberg Gas, Phys. Rev. Lett. 113, 023006 (2014).

[13] H. Schempp, G. Günter, M. Robert-de Saint-Vincent, C. S. Hofmann, D. Breyel, A. Komnik, D. W. Schönleber, M. Gärttner, J. Evers, S. Whitlock, and M. Weidemüller, Full Counting Statistics of Laser Excited Rydberg Aggregates in a OneDimensional Geometry, Phys. Rev. Lett. 112, 013002 (2014).

[14] A. Urvoy, F. Ripka, I. Lesanovsky, D. Booth, J. P. Shaffer, T. Pfau, and R. Löw, Strongly Correlated Growth of Rydberg Aggregates in a Vapor Cell, Phys. Rev. Lett. 114, 203002 (2015).

[15] T. M. Weber, M. Honing, T. Niederprum, T. Manthey, O. Thomas, V. Guarrera, M. Fleischhauer, G. Barontini, and H. Ott, Mesoscopic Rydberg-blockaded ensembles in the superatom regime and beyond, Nat. Phys. 11, 157 (2015).

[16] R. Löw, H. Weimer, J. Nipper, J. B. Balewski, B. Butscher, H. P. Büchler, and T. Pfau, An experimental and theoretical guide to strongly interacting Rydberg gases, J. Phys. B 45, 113001 (2012).

[17] T. E. Lee, H. Häffner, and M. C. Cross, Antiferromagnetic phase transition in a nonequilibrium lattice of Rydberg atoms, Phys. Rev. A 84, 031402 (2011). 
[18] J. Honer, R. Löw, H. Weimer, T. Pfau, and H. P. Büchler, Artificial Atoms Can Do More Than Atoms: Deterministic Single Photon Subtraction from Arbitrary Light Fields, Phys. Rev. Lett. 107, 093601 (2011).

[19] A. W. Glaetzle, R. Nath, B. Zhao, G. Pupillo, and P. Zoller, Driven-dissipative dynamics of a strongly interacting Rydberg gas, Phys. Rev. A 86, 043403 (2012).

[20] C. Ates, B. Olmos, J. P. Garrahan, and I. Lesanovsky, Dynamical phases and intermittency of the dissipative quantum Ising model, Phys. Rev. A 85, 043620 (2012).

[21] M. Lemeshko and $H$. Weimer, Dissipative binding of atoms by non-conservative forces, Nat. Commun. 4, 2230 (2013).

[22] A. Hu, T. E. Lee, and C. W. Clark, Spatial correlations of onedimensional driven-dissipative systems of Rydberg atoms, Phys. Rev. A 88, 053627 (2013).

[23] M. Höning, D. Muth, D. Petrosyan, and M. Fleischhauer, Steadystate crystallization of Rydberg excitations in an optically driven lattice gas, Phys. Rev. A 87, 023401 (2013).

[24] J. Otterbach and M. Lemeshko, Dissipative Preparation of Spatial Order in Rydberg-Dressed Bose-Einstein Condensates, Phys. Rev. Lett. 113, 070401 (2014).

[25] J. Sanders, R. van Bijnen, E. Vredenbregt, and S. Kokkelmans, Wireless Network Control of Interacting Rydberg Atoms, Phys. Rev. Lett. 112, 163001 (2014).

[26] M. Hoening, W. Abdussalam, M. Fleischhauer, and T. Pohl, Antiferromagnetic long-range order in dissipative Rydberg lattices, Phys. Rev. A 90, 021603 (2014).

[27] M. Marcuzzi, E. Levi, S. Diehl, J. P. Garrahan, and I. Lesanovsky, Universal Nonequilibrium Properties of Dissipative Rydberg Gases, Phys. Rev. Lett. 113, 210401 (2014).

[28] H. Weimer, Variational Principle for Steady States of Dissipative Quantum Many-Body Systems, Phys. Rev. Lett. 114, 040402 (2015).

[29] E. Süli and D. F. Mayers, An Introduction to Numerical Analysis (Cambridge University Press, Cambridge, 2003).

[30] F. W. G. Transchel, A. Milsted, and T. J. Osborne, A Monte Carlo time-dependent variational principle, arXiv:1411.5546 [quant$\mathrm{ph}]$.

[31] J. Cui, J. I. Cirac, and M. C. Bañuls, Variational Matrix Product Operators for the Steady State of Dissipative Quantum Systems, Phys. Rev. Lett. 114, 220601 (2015).

[32] E. Mascarenhas, H. Flayac, and V. Savona, Matrix-productoperator approach to the nonequilibrium steady state of driven-dissipative quantum arrays, Phys. Rev. A 92, 022116 (2015).

[33] A. Tomadin, V. Giovannetti, R. Fazio, D. Gerace, I. Carusotto, H. E. Türeci, and A. Imamoglu, Signatures of the superfluidinsulator phase transition in laser-driven dissipative nonlinear cavity arrays, Phys. Rev. A 81, 061801 (2010).
[34] D. Poletti, P. Barmettler, A. Georges, and C. Kollath, Emergence of Glasslike Dynamics for Dissipative and Strongly Interacting Bosons, Phys. Rev. Lett. 111, 195301 (2013).

[35] I. Vidanović, D. Cocks, and W. Hofstetter, Dissipation through localized loss in bosonic systems with long-range interactions, Phys. Rev. A 89, 053614 (2014).

[36] H. Weimer, Variational analysis of driven-dissipative Rydberg gases, Phys. Rev. A 91, 063401 (2015).

[37] M. F. Maghrebi and A. V. Gorshkov, Nonequilibrium manybody steady states via Keldysh formalism, arXiv:1507.01939 [cond-mat.quant-gas].

[38] J. J. Mendoza-Arenas, S. R. Clark, S. Felicetti, G. Romero, E. Solano, D. G. Angelakis, and D. Jaksch, Beyond mean-field bistability in driven-dissipative lattices: bunching-antibunching transition and quantum simulation, arXiv:1510.06651 [quant$\mathrm{ph}$.

[39] J. Johansson, P. Nation, and F. Nori, QuTiP. 2: A Python framework for the dynamics of open quantum systems, Comput. Phys. Commun. 184, 1234 (2013).

[40] M. J. W. Hall, J. D. Cresser, L. Li, and E. Andersson, Canonical form of master equations and characterization of non-Markovianity, Phys. Rev. A 89, 042120 (2014).

[41] A. Rivas, S. F. Huelga, and M. B. Plenio, Quantum nonMarkovianity: characterization, quantification and detection, Rep. Prog. Phys. 77, 094001 (2014).

[42] V. Gorini, A. Kossakowski, and E. C. G. Sudarshan, Completely positive dynamical semigroups of N-level systems, J. Math. Phys. 17, 821 (1976).

[43] R. M. Angelo, S. A. Vitiello, M. A. M. de Aguiar, and K. Furuya, Quantum linear mutual information and classical correlations in globally pure bipartite systems, Physica A 338, 458 (2004).

[44] C. Kollath, A. M. Läuchli, and E. Altman, Quench Dynamics and Nonequilibrium Phase Diagram of the Bose-Hubbard Model, Phys. Rev. Lett. 98, 180601 (2007).

[45] S. R. Manmana, S. Wessel, R. M. Noack, and A. Muramatsu, Strongly Correlated Fermions after a Quantum Quench, Phys. Rev. Lett. 98, 210405 (2007).

[46] S. Trotzky, Y.-A. Chen, A. Flesch, I. P. McCulloch, U. Schollwock, J. Eisert, and I. Bloch, Probing the relaxation towards equilibrium in an isolated strongly correlated one-dimensional Bose gas, Nat. Phys. 8, 325 (2012).

[47] M. Rigol, V. Dunjko, and M. Olshanii, Thermalization and its mechanism for generic isolated quantum systems, Nature (London) 452, 854 (2008).

[48] T. Langen, S. Erne, R. Geiger, B. Rauer, T. Schweigler, M. Kuhnert, W. Rohringer, I. E. Mazets, T. Gasenzer, and J. Schmiedmayer, Experimental observation of a generalized Gibbs ensemble, Science 348, 207 (2015).

[49] P. Doria, T. Calarco, and S. Montangero, Optimal Control Technique for Many-Body Quantum Dynamics, Phys. Rev. Lett. 106, 190501 (2011). 